\title{
Einsatz des Computers im Geographieunterricht
}

\author{
Kurt Brassel
}

\section{Einleitung}

Im Wintersemester 1970/71 wurde im Rahmen der Kartenkundeübungen des Geographischen Instituts der Universität Zürich erstmals der Computer als zentrales Hilfsmittel für ein Übungsbeispiel eingesetzt. Da dieser Versuch gelungen ist, wollen wir hier einen Überblick über Konzeption und Durchführung dieser Übung geben.

In weiten Kreisen fühlt man sich einerseits etwas unsicher gegenüber der elektronischen Datenverarbeitung, und andererseits werden die Rechenautomaten wieder überschätzt. Es ist deshalb mit ein Anliegen dieses Artikels, einen Einblick in das Arbeiten mit Computern zu geben und mögliche Arbeitsbereiche für dieses Hilfsmittel aufzuzeigen.

Sind die Voraussetzungen für die Durchführung von Ưbungen im vorgeschlagenen Stil vorerst vielleicht nur für Hochschulen gegeben, so darf doch darauf hingewiesen werden, daß auch einzelne Mittelschulen (z. B. Zürcher Kantonsschulen) zu Computern Zugang haben und sie benützen. - Auch betreiben verschiedene kantonale Verwaltungen Datenverarbeitungsanlagen, so daß bei Bedarf eventuell auch anderen Kantonsschulen entsprechende Möglichkeiten offenstünden.

Die ursprüngliche Bedeutung des Computers liegt in der Fähigkeit, genau und rasch beliebige Berechnungen durchzuführen. Vermehrt findet er aber auch für andere Zwecke Anwendung, und wir haben ihn vorwiegend dazu benutzt, mit dem Schnelldrucker (Printer) statistische Karten herzustellen.

Das Ausdrucken von Karten mit dem Computer bringt einige Vorteile mit sich; so wird die handwerkliche Arbeit auf ein Minimum herabgesetzt. Außerdem bietet es die Möglichkeit, mit den Abbildungen $\mathrm{zu}$ experimentieren, indem nach einem ersten Darstellungsversuch der Output begutachtet und korrigiert wird und mit geringem Aufwand wieder eine neue Karte erstellt werden kann. Liegen verschiedene Datensätze vor, so hat man die Möglichkeit, diese beliebig zu korrelieren und die Resultate wieder graphisch darzustellen.

Diesen Vorteilen stehen aber auch Nachteile gegenüber: Die Abbildungen aus dem Schnelldrucker sind an das rechteckige Maschennetz der Druckpositionen gebunden, und es muß mit Ungenauigkeiten in bezug auf Zwischenräume und Schwär- zung gerechnet werden. Die produzierten Karten sind also in ihrer Qualität beschränkt, doch eignen sie sich unbestritten als Arbeitskarten. Ferner muß darauf hingewiesen werden, $d a ß$ in solchen Úbungen die handwerkliche Schulung des Kartenzeichnens dahinfällt. Demgegenüber tritt aber die gedankliche Arbeit in den Vordergrund, und das Erstellen von automatisierten Darstellungen ist deshalb nicht als Ersatz, sondern vielmehr als Ergänzung der Ausbildung zu betrachten.

\section{Übungsgrundlagen}

Beim hier gezeigten Anwendungsbeispiel handelt es sich um folgende Aufgabe:

Aus statistischen Werten der Gemeinden des Kantons Zürich soll eine Choroplethenkarte mit maximal 10 Klassen erstellt werden. Die Karte wird mit dem Computer durch Übereinanderschlagen von 0 bis 4 Signaturen ausgedruckt; die benötigten Programme sowie weitere Unterlagen liegen bereits vor (durch Assistenten vorbereitet). Die Studenten haben folgende Arbeiten durchzuführen:

- Auswahl des darzustellenden Themas

- Ablochen der statistischen Daten

- Festsetzen der Klassengrenzen

- Auswählen der Signaturen oder Signaturkombinationen für die einzelnen Klassen.

Die restlichen Arbeiten werden durch die Maschine geleistet.

In didaktischer Hinsicht werden mit dieser Úbung folgende Ziele verfolgt:

- Schulung der Bearbeitung von Choroplethenkarten: Klassenbildung, Graustufenwahl, Signaturwahl

- Verwendung von Diagrammen als Hilfsmittel; Interpretation der Diagramme

- Einblick in die Grundprinzipien der Datenverarbeitung: Programme - Dateien, Erstellen eines Datensatzes, Kenntnis des Lochkartenlochers.

Die Úbung baut im wesentlichen auf bereits bekannten und beschriebenen Techniken auf (s. Literatur- 


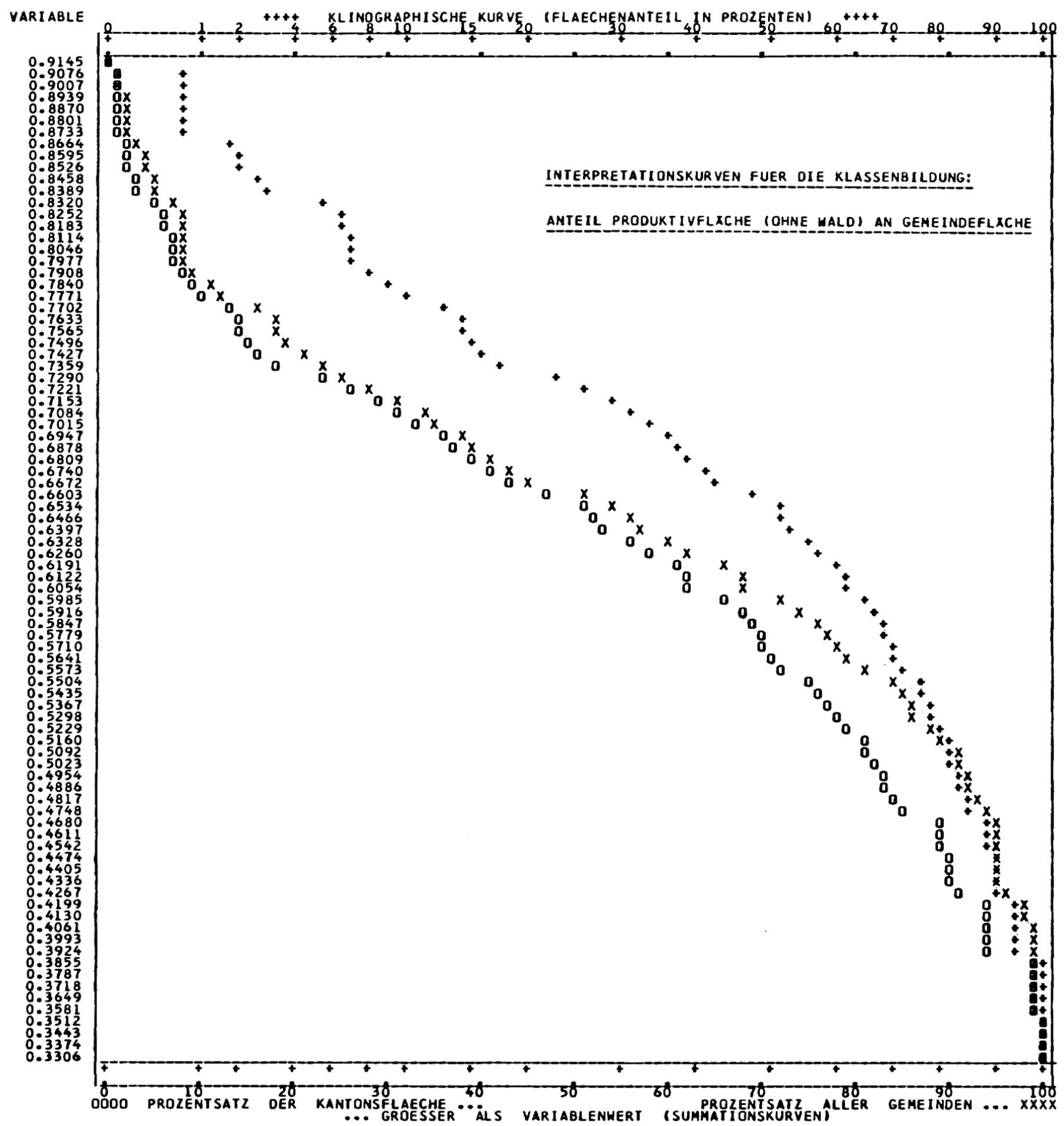

Figur 1

hinweise); es wird lediglich versucht, diese mit neuen Hilfsmitteln durchzuführen.

In Choroplethenkarten, die an statistische Einheiten gebunden sind, stellen wir die Daten (besonders geeignet sind Verhältniszahlen, zum Beispiel Dichtewerte) in einfacher Weise dar: Den Grundflächen der statistischen Zähleinheiten (Gemeinden, Bezirke) wird der entsprechende statistische Wert als dritte Dimension zugeordnet (horizontale Fläche). Damit bei der Darstellung nicht für jede statistische
Einheit eine spezielle Signatur gesetzt werden muß, wird das Spektrum der statistischen Werte in eine Zahl von Klassen unterteilt und den einzelnen Klassen eine Signatur zugewiesen. Da die Klassengrenzen an beliebiger Stelle gesetzt werden können, besteht das Hauptproblem unserer Úbung darin, diese Begrenzungen richtig zu setzen; also so, daß einerseits der wahre Sachverhalt nicht verfälscht wird und andererseits die Darstellung graphisch befriedigend ausfällt. Die Wahl der Klassen kann aber 


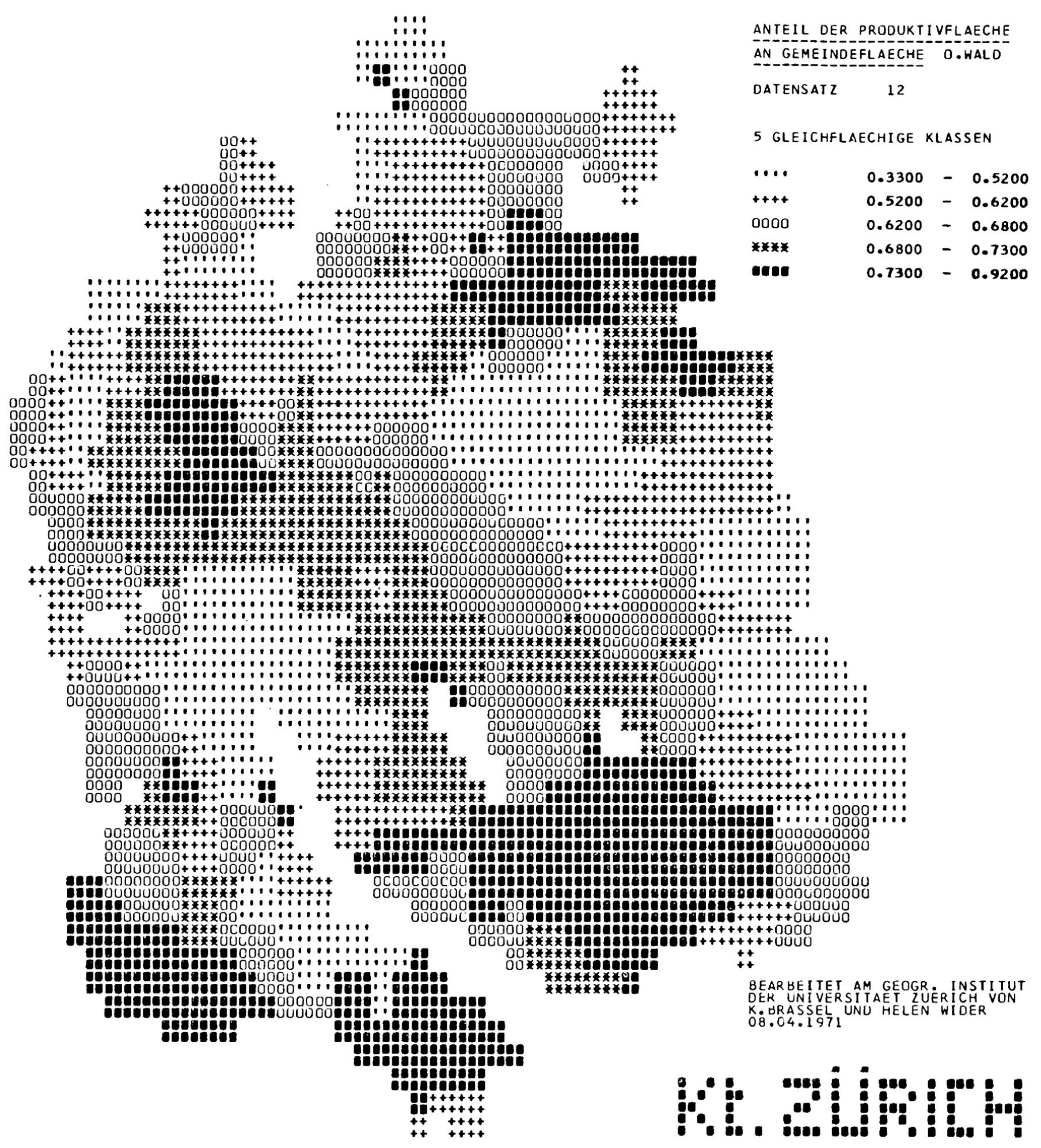

Figur 2. Beispiel einer mit dem Computer erstellten statistischen Karte des Kantons Zürich

nur dann sinnvoll erfolgen, wenn man die Häufigkeitsverteilung der Variablen über das ganze Spektrum zwischen Minimal- und Maximalwert kennt. Wir erstellen uns deshalb mit dem Computer verschiedene Interpretationskurven (Klinogramme, Verteilungskurven, s. Fig. 1), die das Setzen der Klassengrenzen erleichtern. In einem zweiten Schritt lassen wir dann den Computer eine entsprechende Karte ausdrucken (Fig. 2).

Die Computerarbeiten wurden im Rechenzentrum der Universität Zürich auf einer IBM/360-50, die Hilfsprogramme zum Teil auf einer IBM 1620 durchgeführt.

\section{Durchführung der Übung}

Wir haben den Ablauf der Übungen in Form eines Blockdiagramms schematisch dargestellt (s. Figur 


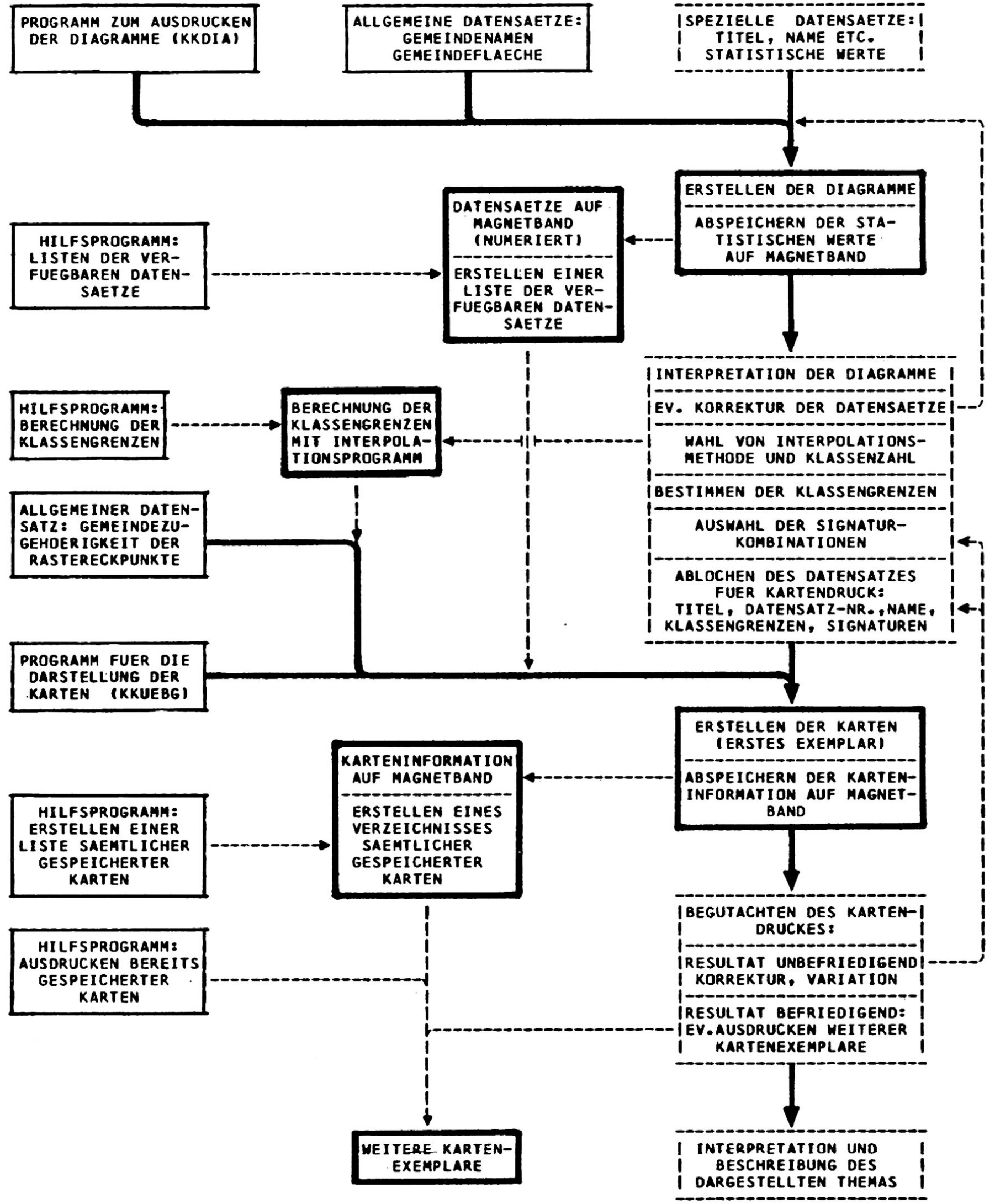

Figur 3. Uberblick über den Übungsablauf

(fette Rahmen: Vorgänge im Computer; gestrichelte Rahmen: Bearbeitung durch Studenten; feine Umrahmung: Vorbereitungsarbeiten durch Assistenten; Pfeile: Flußrichtung, unterschieden nach Wichtigkeit)

3). Die einzelnen Kästchen stellen Arbeitsschritte dar, wobei der Gesamtablauf im wesentlichen von oben nach unten verläuft. Aus der Umrandung wird ersichtlich, durch wen die entsprechende
Handlung ausgeführt wird. Wir unterscheiden zwischen der Arbeit der Studenten, zwischen Assistenzarbeit und den Berechnungen und Manipulationen durch den Computer. 
Als eigentliche UUbungsvorbereitung waren neben rein administrativen Belangen einige allgemeine Datensätze (rechteckiges Rasternetz über der Kantonsfläche, Gemeindezugehörigkeit der Rasterpunkte usw.) bereitzustellen. Ferner waren einige Programme zu entwerfen und auszutesten, nämlich die beiden Hauptprogramme KKDIA (Diagrammdarstellung) und KKUEBG (Kartendruck) sowie verschiedene Hilfsprogramme, die zur Organisation und Manipulation der Datensätze gebraucht wurden.

Die Studenten hatten vorerst je einen statistischen Datensatz abzulochen (Gemeindedaten des Kantons Zürich, 20 bzw. 40 Lochkarten; ein Lochkartenstanzer dazu steht im Geographischen Institut zur Verfügung). Die Kartenpakete der Studenten wurden eingesammelt und mit dem entsprechenden Programm in logisch richtiger Reihenfolge dem Rechenautomaten übergeben.

Für jeden eingegebenen Datensatz druckte nun der Computer ein Diagramm (Fig. 1) aus. Es sind darin eine klinographische Kurve (Legende oben: +-Signatur) sowie zwei Verteilungskurven (Legende unten) dargestellt. Die eine (X-Signatur) berücksichtigt die Gemeindezahl, sagt also aus, welcher Prozentanteil sämtlicher Zürcher Gemeinden über einem bestimmten Variablenwert liegt, wobei dieser Wert vom Maximum zum Minimum variiert wird. Die andere (O-Signatur) hält den Anteil an der gesamten Kantonsfläche fest, den die Gemeinden über einem jeweiligen Variablenwert ausmachen. Dasselbe gilt für die klinographische Kurve, nur basiert sie auf einer nichtlinearen Skala (näheres s. Jenks und Coulson 1963, S. 126). Die beiden letztgenannten Kurven sind für die Interpretation, die dann von den Studenten vorgenommen wurde, von besonderer Bedeutung. Beim Setzen der Klassengrenzen kann mit ihrer Hilfe abgeschätzt werden, welcher Anteil der dargestellten Kantonsfläche in eine betreffende Klasse fällt, also mit einer bestimmten Signatur versehen wird.

Die Klassen können nun nach verschiedenen Kriterien abgegrenzt werden: Entweder legt man die Klassengrenzen auf Knickstellen der klinographischen Kurve, an Steilstufen der Verteilungskurven, oder man unterteilt die Abszisse (\%-Anteil) in gleiche Abschnitte (flächengleiche Klassen). Entspricht der Verlauf der Verteilungskurven dem Graph einer mathematischen Funktion (z. B. arithmetisch oder geometrisch wachsender Verlauf; s. Jenks, 1967), so können, falls Klassenzahl sowie Ober- und Untergrenzen der Variablen bekannt sind, die Klassengrenzen berechnet werden. Für diese Berechnungen wurde ein Hilfsprogramm verfertigt, das von den Studenten bei Bedarf verwendet werden konnte.

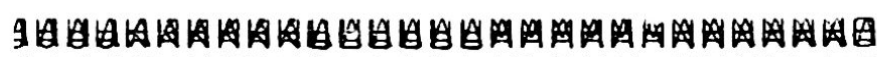
J J J JKKKKKKLLLLLLMMMNIMIVINNNNNINC

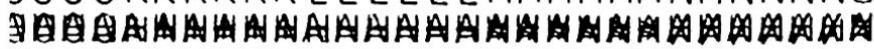

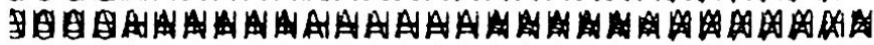

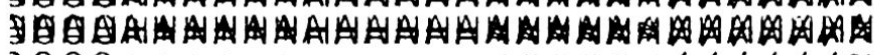
$3000++++++--\cdots---\# \# \# \# / 1 / 1 / 1 \%$

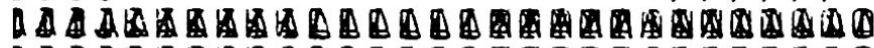

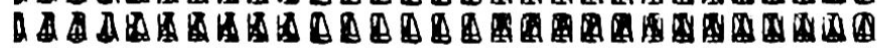
A JJJJKKKKKKLLLLLLMMMMMMNNNNIVNO

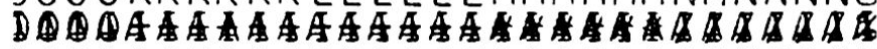

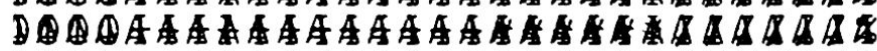

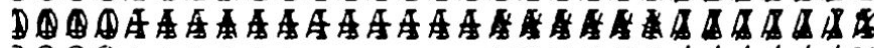

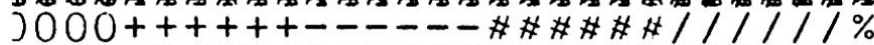

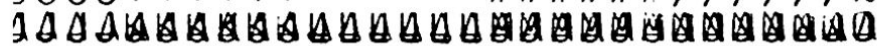

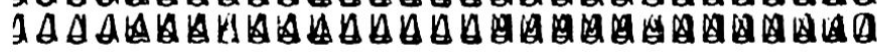

Figur 4. Ausschnitt aus dem Signaturenkatalog: Übereinanderdruck von je 3 Zeichen

Liegt die Klassenzahl vor und sind die Klassengrenzen festgelegt, so ist für jede Klasse eine Signaturkombination (0-4 Zeichen übereinander) auszuwählen. Für diese Auswahl steht ein Katalog, der 3 fache Übereinanderdrucke sämtlicher möglicher Signaturen enthält (s. Fig. 4), zur Verfügung.

Ist auch diese Arbeit erledigt, so werden Titel, Datensatznummer, Name, Klassengrenzen und Signaturen (total 4 Lochkarten) abgelocht und die Karten mit Hilfe des Programms KKUEBG ausgedruckt (Fig. 2). Hat sich ein Fehler eingeschlichen oder ist eine Signatur nicht sachgerecht gesetzt, so kann durch Korrektur der entsprechenden Lochkarte eine verbesserte Karte hergestellt werden. Es ist auch möglich, durch Variation der Klassenzahl oder der Klassenbildungskriterien Vergleichsreihen von Karten ausdrucken zu lassen.

Entsprechend der Aufteilung der Arbeitsschritte war der Zeitplan der Übung flexibel zu gestalten. Nach einer einführenden Orientierung und dem anfänglichen Ablochen des Datensatzes hatten die Studenten rund einen Monat Zeit bis zur Abgabe von Kommentaren zum dargestellten Thema. Dabei beschränkte sich die Arbeit im wesentlichen auf Interpretation von Diagramm und Karten. Die Jobs konnten wöchentlich zweimal abgegeben werden. Anhand dieser Bedingungen war es den Studenten möglich, ohne viel handwerklichen Aufwand sowohl die Kartendrucke immer wieder zu verbessern als auch die Problemstellung der Übung, die Klassenbildung, zu vertiefen.

Um dem Leser einen Einblick in die Funktionsweise der beiden Hauptprogramme zu geben, seien am Schluß die wichtigsten Programmschritte stichwortartig zusammengestellt: 
Programm zum Ausdrucken der Diagramme (KKDIA)

a) Einlesen der allgemeinen Daten: Gemeindenamen, Gemeindefläche usw.

b) Einlesen der speziellen Daten: Titel, Name des Studenten, statistische Variablenwerte

c) Modifikation und Speichern der Variablenwerte auf Magnetband

d) Sortieren der Gemeinden nach der Größe der Variablenwerte

e) Ausdrucken der sortierten Variablenwerte mit Gemeindenamen

f) Berechnung der Diagrammskala in Ordinatenrichtung (s. Abb. 1) aus Maximal- und Minimalwert der darzustellenden Variablen und aus der Gesamthöhe des Diagramms (Zeilenzahl)

g) Berechnung der Abszissenwerte der Kurven (Prozentanteile); für jede Diagrammzeile muß je ein Wert für die klinographische und die Verteilungskurven bestimmt werden

h) Ausdrucken des Diagramms inklusive Titel, Datensatznummer und Name

i) Sprung zurück zu b) (Einlesen der Daten des nächsten Studenten)

\section{KKUEBG: Programm zum Ausdrucken der Karten}

a) Einlesen der allgemeinen Daten: Gemeindezugehörigkeit der einzelnen Rasterpunkte

b) Einlesen der speziellen Daten: Titel, DatensatzNr., Name des Studenten, Signaturkombinationen, Klassengrenzen; statistische Daten (ab Magnetband)

c) Zuordnen einer Signaturkombination für jeden Rasterpunkt:

Der Rasterpunkt

fällt auf die Gemeinde

Der Variablenwert der betreffenden Gemeinde beträgt

er liegt also zwischen den

beiden Klassengrenzen

Der betreffende Rasterpunkt

fällt also in die Klasse 3

und somit wird ihm folgende $-\mathrm{X}$

Signaturkombination zugeordnet (übereinander)
Jeder Rasterpunkt des darzustellenden Gebietes (von $1 / 1$ bis 60/48) wird nach diesem Schema bearbeitet.

d) Zusammenstellen der Abbildung im Hauptspeicher: Plazieren von Titel, Legende, Namen, Daten usw. an logisch richtiger Stelle

e) Ausdrucken der Karte

f) Abspeichern des logisch geordneten Karteninhaltes auf Magnetband (für allfällige weitere Kartenexemplare)

g) Sprung zurück zu b) (Einlesen der Daten des nächsten Studenten)

Die Datensätze werden also im Stapel eingegeben und in Serie bearbeitet. Ist in einem Datensatz eines Studenten irgendwo ein Fehler eingelocht, so gibt das Programm eine Fehlermeldung heraus und springt direkt wieder zum Ausgangspunkt b). - Für einen Durchgang des Programmes KKUEBG (Schritte b bis g) braucht die IBM/360-50 rund 90 Sekunden.

\section{Literaturhinweise}

Jenks G. und Coulson R.: Class Intervals for Statistical Maps, Int. Jahrbuch für Kartographie, III/ 1963, S. 119-134.

Jenks G. F.: The Data Model Concept in Statistical Mapping, Int. Jahrbuch für Kartographie, Band VII, S. 186-190.

Kishimoto H. und Boesch H.: Einige Bemerkungen zur kartographischen Ausbildung von Geographen, in «K+F-Kreis», 12. Jahrgang, Nr. 24, Bern, Mai 1971.

Mackay J. R.: An Analysis of Isopleth and Choropleth Class Intervals, in Econ. Geogr. 1/1955, Clark University Worcester, USA. 